\title{
Tilt Angle Adaptation in LTE Networks with Advanced Interference Mitigation
}

\author{
Bahar Partov ${ }^{1}$, Douglas J. Leith ${ }^{1}$, and Rouzbeh Razavi ${ }^{2}$ \\ ${ }^{1}$ Hamilton Institute, NUI Maynooth, ${ }^{2}$ Bell Laboratories, Alcatel-Lucent, Dublin
}

\begin{abstract}
In this paper we investigate the role of tilt angle adjustment in next generation LTE networks where SIMO receivers and adaptive OFDM/TDMA transmission scheduling may additionally be used to mitigate interference. We present detailed performance measurements when (i) optimal fair tilt angle adjustment is applied in combination with SIMO receivers using Linear Minimum Mean Square Error (LMMSE) detection to mitigate interference, and when (ii) tilt angle adjustment is applied in combination with proportional fair OFDM transmission scheduling which adapts the transmission rate per subcarrier (narrow-band rate allocation). We find that even when SIMO/LMMSE reception and adaptive transmission scheduling are used to mitigate interference, tilt angle adjustment still offers the potential for significant performance gains, namely increases in mean user throughput of more than $65 \%$ and improvements in the network sum-log rate of greater than $20 \%$.
\end{abstract}

Index Terms-Antenna tilt angle, LTE, Proportional fairness, LMMSE detection, Multi-user diversity

\section{INTRODUCTION}

The antenna tilt angle of wireless base-stations is known to impact cell coverage and interference management in existing cellular networks [1], [2]. In this paper we investigate the role of tilt angle adjustment in next generation LTE networks where SIMO receivers and adaptive OFDM/TDMA transmission scheduling may additionally be used to mitigate interference [3]. Receiver handsets employing two antennas, which are anticipated to become the norm, can use the additional singleinput multiple-output (SIMO) reception diversity to cancel a primary interferer and so boost effective (i.e. post-processing) SINR. Modern basestations can adaptively schedule transmissions amongst subcarriers and across time slots and so, in particular, can schedule transmissions to mitigate intercell interference. Since tilt angle adjustment also impacts intercell interference, the interaction between tilt angle adjustment, SIMO detection and adaptive scheduling is of considerable interest.

We build upon a recently introduced approach [4] for proportional fair tilt angle adjustment. In [4] we show that although this optimisation problem is non-convex, under certain conditions it can be re-formulated as a convex problem which can be solved efficiently using information which is already available at base stations. For a realistic network configuration we present detailed performance measurements where this proportional fair tilt angle adjustment is applied

This material is based upon works supported by the Science Foundation Ireland under Grant No. 11/PI/1177 and by Bell Labs Ireland.

978-1-4799-4912-0/14/\$31.00 (C)2014 IEEE in combination with SIMO receivers using Linear Minimum Mean Square Error (LMMSE) detection to mitigate interference. We also present detailed measurements when tilt angle adjustment is applied in combination with proportional fair OFDM transmission scheduling which adapts the transmission rate per subcarrier (narrow-band rate allocation) to exploit multi-user diversity and mitigate interference. We find that even when SIMO/LMMSE reception and adaptive transmission scheduling are used to mitigate interference, tilt angle adjustment still offers the potential for significant performance gains. Of particular interest is that these performance gains are achieved even when the tilt angle adjustment assumes basic single antenna receivers and non-adaptive round-robin frequency scheduling. That is, there appears to exist a useful degree of decoupling between tilt angle adjustment and the details of receiver and transmitter design which has the potential to greatly simplify network design.

The rest of the paper is organized as follows. In Section II we summarize the existing work in the area. In Section III we introduce our network model, which is based on 3GPP standard. In Section IV we consider the performance of SIMO receivers with LMMSE detection when tilt angle optimisation is used, then in Section $\mathrm{V}$ we consider the performance when adaptive proportional fair OFDM transmit scheduling is used together with tilt angle optimisation. Finally, in Section VI, we summarise our conclusions.

\section{RELATED WORK}

The analysis and modelling of the impact of the antenna tilt angle on cell performance has been well studied, see for example [5], [6] and references therein. Recently, self-optimisation of tilt angle has started to attract attention, but most of this work makes use of heuristic approaches. In [7] a heuristic method is proposed for adjusting tilt to maximise average spectral efficiency within the network, while [8] proposes a combination of fuzzy and reinforcement learning. In [9] simulated annealing is considered for joint self-configuration of antenna tilt angle and power and in [10] a non-cooperative game approach between neighbouring base stations is studied. Offline planning of tilt angle is considered, for example, in [11], using a heuristic search method combined with a mixed integer local search. However, none of them consider other interference mitigation techniques particularly techniques which are mentioned in Section I in conjunction with the optimisation task, or neither have evaluated the proposed solutions while other interference mitigation techniques are present. In the 
present paper, we extend our work in [4] by evaluating other contributing factor in interference management.

\section{PRELIMINARIES}

\section{A. Network Model}

1) Network Architecture: The network consists of a set $\mathcal{B}$ of base stations and a set $\mathcal{U}$ of User Equipment (UE), with UE $u \in \mathcal{U}$ receiving downlink traffic transmitted from base station $b(u) \in \mathcal{B}$. For base stations with sectoral antennas, we define a separate element in $\mathcal{B}$ for each antenna. We denote the $(x, y)$ geographical co-ordinates of base station $b$ by $\left(x_{b}, y_{b}\right)$ and of user equipment $u$ by $\left(x_{u}, y_{u}\right)$. The distance between user $u$ and base station $b$ is therefore given by

$$
d_{b, u}=\sqrt{\left(x_{u}-x_{b}\right)^{2}+\left(y_{u}-y_{b}\right)^{2}}
$$

2) Antenna Gain and Path Loss: The received power on sub-carrier $n$ from base station $b \in \mathcal{B}$ at user $u \in \mathcal{U}$ is given by $\tilde{G}_{b, u}\left(\theta_{b}\right) \rho_{b, u} P_{b, n}$, where $\tilde{G}_{b, u}\left(\theta_{b}\right)$ is the base station antenna gain, $\rho_{b, u}$ the path loss between $b$ and $u, P_{b, n}$ is the base station transmit power for sub-carrier $n$. For simplicity, shadowing and fast fading are not considered in the equations. We model path loss, as recommended in [12], by

$$
\rho_{b, u}=\rho_{0} d_{b, u}^{-\beta}
$$

with fixed path loss factor $\rho_{0}$, path loss exponent $\beta$ and distance $d$ in kilometres. For a given antenna type, the antenna gain $\tilde{G}_{b, u}\left(\theta_{b}\right)$ can be determined given the relative positions of $b$ and $u$, the antenna tilt angle $\theta_{b}$ and the azimuth angle $\phi_{b}$. With regard to the latter, changing the tilt and/or azimuth angles changes the direction of the antenna's main lobe. We will assume that the azimuth angle is held fixed but allow the antenna tilt angle to be adjusted within the interval $[\underline{\theta}, \bar{\theta}]$. Following [12], the antenna gain can then be modelled by:

$$
\tilde{G}_{b, u}\left(\theta_{b}\right)=\tilde{G}_{0} \tilde{G}_{v}\left(\theta_{b}, d_{b, u}\right)
$$

where $\tilde{G}_{0}$ is the maximum gain of the antenna,

$$
\tilde{G}_{v}\left(\theta_{b}, d_{b, u}\right)=10^{-1.2\left(\frac{\theta_{b, u}-\theta_{b}}{\theta_{3 d B}}\right)^{2}}
$$

is the antenna vertical attenuation, $\theta_{b, u}=\tan ^{-1}\left(h / d_{b, u}\right), h$ is the height difference between the base station and UE (which, for simplicity, we assume is the same for all base stations and users) and $\theta_{3 d B}$ the vertical half power beam width of the antenna. Figure 1 illustrates the ability of (4) to accurately model the main lobe of an antenna which is popular in cellular networks.

We define $G_{v}\left(\theta_{b}, d_{b, u}\right):=\log \tilde{G}_{v}\left(\theta_{b}, d_{b, u}\right)$. We also define the following linear approximation $\hat{G}_{v}\left(\theta_{b}, d_{b, u}\right)$ to antenna gain exponent $G_{v}\left(\theta_{b}, d_{b, u}\right)$ about $\theta_{0}$ tilt angle,

$$
\hat{G}_{v}\left(\theta_{b}, d_{b, u}\right)=\frac{-1.2 \log 10}{\theta_{3 d B}^{2}}\left(\left(\theta_{b, u}-\theta_{0}\right)^{2}+2\left(\theta_{b, u}-\theta_{0}\right) \theta_{b}\right)
$$

which we show in [4] is a reasonably accurate approximation for inter-cell interference.

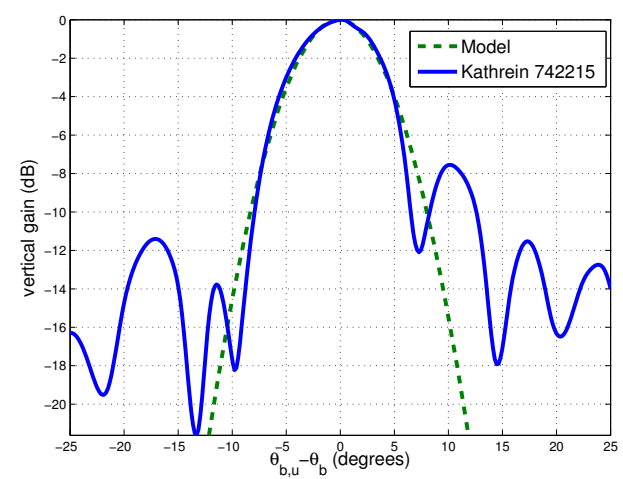

Fig. 1. Comparison of antenna main lobe vertical gain model (4) (dashed line) and measured antenna gain (solid line) for a Kathrein 742215 antenna, $\theta_{3 d B}=9^{\circ}$.

\section{B. Tilt Angle Adjustment}

Following [4], we select the basestation antenna tilt angles that maximise the following utility fair optimisation $P$ :

$$
\begin{array}{ll}
\max _{\Theta} & \sum_{u \in \mathcal{U}} \log R_{u}(\Theta) \\
\text { s.t. } \quad & \underline{\theta} \leq \theta_{b} \leq \bar{\theta}, \quad b \in \mathcal{B} \\
& \log \underline{r} \leq \log R_{u}(\Theta), \quad u \in \mathcal{U}
\end{array}
$$

where the downlink throughput $R_{u}(\Theta)$ of the user equipment $u \in \mathcal{U}$ associated with base station $b(u)$ is given by:

$$
R_{u}(\Theta)=\min \left\{\bar{r}, r_{u}(\Theta)\right\}, \quad u \in \mathcal{U}
$$

with $\Theta$ the vector $\left[\theta_{b}\right], b \in \mathcal{B}$ of tilt angles, $\bar{r}$ the maximum achievable throughput (limited by the available modulation and coding schemes), and

$$
r_{u}(\Theta)=\frac{w}{N_{s c}} \sum_{n=1}^{N_{s c}} \log \left(1+\kappa \gamma_{u, n}(\Theta)\right)
$$

Here $N_{s c}$ is the number of sub-carriers, $w$ the channel bandwidth, $\kappa$ a loss factor capturing non-ideal coding etc, and $\gamma_{u, n}(\Theta)$ SINR on sub carrier $n$ for user $u$,

$$
\gamma_{u, n}(\Theta)=\frac{H_{u}\left(\theta_{b(u)}\right)}{\sum_{c \in \mathcal{B} \backslash\{b(u)\}} \hat{H}_{u}(c)+\eta_{u, n}}
$$

where $H_{u}\left(\theta_{b}\right):=e^{G_{b, u}\left(\theta_{b}\right)} \rho_{b, u} P_{b, u}$ is the received power from base station $b(u)$ by user $u, \hat{H}_{u}\left(\theta_{c}\right):=e^{\hat{G}_{c, u}\left(\theta_{c}\right)} \rho_{c, u} P_{c, u}$ is the received power from base station $c \neq b(u)$ by user $u$ and $\eta_{u, n}$ the channel noise for user $u$ on sub carrier $n$. Observe that in $\hat{H}_{u}\left(\theta_{c}\right)$ we make use of linear approximation $\hat{G}_{v}$.

In [4] we have shown that this optimisation problem is nonconvex, but under reasonable conditions it can be reformulated as a convex optimisation and the optimal tilt angles efficiently calculated.

\section{LTE SIMO RECEIVERS}

In this section we consider LTE SIMO links with $1 \mathrm{Tx}$ antenna on the BS and $2 \mathrm{Rx}$ antennas at the UE. The presence of two antennas at the receiver allows the UE to cancel one 
interferer. Hence, if interference is dominated by a single transmitter then we expect the use of SIMO links will allow inter-cell interference to be significantly reduced. Our interest here is in the impact that this interference cancellation has on the size of throughput gain achievable by tilt angle adjustment.

\section{A. MMSE Post-Processing}

We consider LMMSE post processing applied to the received signal to mitigate neighbouring cell interference. Defining channel vector $\mathbf{k}_{u}=\left[\begin{array}{ll}k_{1} & k_{2}\end{array}\right]^{T}$, the channel gain for user $u$ is:

$$
\mathbf{k}_{u}=\sqrt{\frac{H_{u}\left(\theta_{b}\right)}{P}} \sqrt{10^{S_{b, u} / 10}} \mathbf{q}_{b, u} .
$$

where $S$ is a zero mean Gaussian random variable representing slow fading effects, $\mathbf{q}_{b, u}$ is a Rayleigh flat fading vector and $P$ is the power of the transmitted signal assuming all base stations transmit at $P=P_{b, u}$. We can consider the elements of $\mathbf{q}_{b, u}$ to be independent complex random Gaussian processes corresponding to the channels of base station $b$ and user $u$, provided that the antenna elements are sufficiently separated (typically on the order of half a wavelength apart). We identify the inter-cell interference vector $\mathbf{v}_{u}=\left[\begin{array}{ll}v_{1} & v_{2}\end{array}\right]^{T}$ for user $u$ by the strongest interferer:

$$
\mathbf{v}_{u}=\sqrt{\max _{c \in \mathcal{B} \backslash\{b(u)\}}\left\{\hat{H}_{u}\left(\theta_{c}\right) 10^{S_{c, u} / 10}\right\}} \mathbf{q}_{c_{\max }, u}
$$

The remaining inter-cell interference is modelled as spatially white Gaussian noise [13], which comprises the noise vector $\boldsymbol{n}_{u}=\left[\begin{array}{l}n_{1} \\ n_{2}\end{array}\right]$ where $n_{1}$ and $n_{2}$ are independent Gaussian

$$
N_{0}=E\left[n_{1} n_{1}^{H}\right]=E\left[n_{2} n_{2}^{H}\right]=\sum_{\substack{c \in \mathcal{B} \backslash\{b(u)\} \\ c \neq c_{\max }}} \hat{H}_{u}\left(\theta_{c}\right)+\eta_{u}
$$

Hence, the received signal $(\boldsymbol{y})$ is given by:

$$
\mathbf{y}_{u}=\mathbf{k}_{u} \mathbf{x}+\mathbf{v}_{u}+\mathbf{n}_{u}
$$

with $E\left[x x^{H}\right]=P$. The linear MMSE combining vector $\mathbf{w}_{u}=$ $\left[\begin{array}{ll}w_{1} & w_{2}\end{array}\right]^{T}$, is given by:

$$
\mathbf{w}_{u}=\mathbf{k}_{u}^{H}\left(\mathbf{k}_{u} \mathbf{k}_{u}^{H}+\frac{\boldsymbol{\Phi}+N_{0} \mathbf{I}}{P}\right)^{-1}
$$

where $\boldsymbol{\Phi}$ is the autocorrelation of interference vector $\mathbf{v}$ :

$$
\boldsymbol{\Phi}=E\left[\mathbf{v}_{u} \mathbf{v}_{u}^{H}\right]=\left[\begin{array}{cc}
\left|v_{1}\right|^{2} & v_{1} v_{2}^{H} \\
v_{2} v_{1}^{H} & \left|v_{2}\right|^{2}
\end{array}\right]=\left[\begin{array}{ll}
\phi_{11} & \phi_{12} \\
\phi_{21} & \phi_{22}
\end{array}\right]
$$

By applying the MMSE weights on the received signal, the post processing SINR is calculated as:

$$
\begin{aligned}
& \gamma_{u}^{M M S E}= \\
& \left|w_{1}\right|^{2} \phi_{11}+\left|w_{2}\right|^{2} \phi_{22}+2 \operatorname{Re}\left\{w_{1} w_{2}^{H} \phi_{12}\right\}+N_{0}\left(\left|w_{1}\right|^{2}+\left|w_{2}\right|^{2}\right)
\end{aligned}
$$

Post processing SINRs will be averaged over the multipath fading realisations. Using the averaged post processing SINRs,

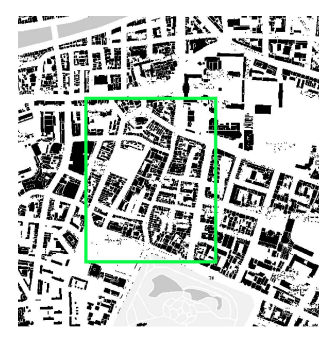

(a) Street map

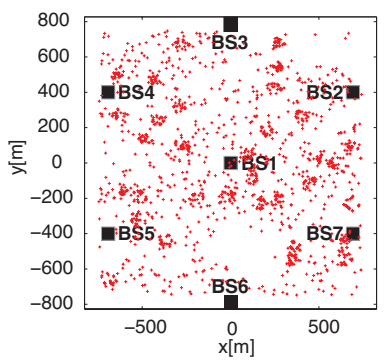

(b) User and base-station locations
Fig. 2. Dublin, Ireland example.

user throughputs can be calculated using (9). Thereafter, solutions to the optimisation problem $P$ can be used to calculate post-processing user throughputs and performance gains can be evaluated.

\section{B. Performance Evaluation}

We consider a realistic example based on data from the cellular network covering Grafton Street and Dawson Street in downtown Dublin, Ireland, see Figure 2a. These are major shopping streets close to the centre of Dublin city, with a large number of cellular users. We consider a section of the network with 21 sectors in a $1500 \mathrm{~m} \times 1500 \mathrm{~m}$ area and with an inter-site distance of $800 \mathrm{~m}$. Environmental characteristics are derived from experimental measurement data with a combination of non-line of sight and line-of sight paths. Path loss and lognormal shadow fading parameters are derived from [12] for macro urban scenarios and detailed Table I. There are 1350 users, with locations as shown in Figure $2 \mathrm{~b}$. We focus on the performance experienced by the 388 users associated with the centre base station (indicated by BS1 in Figure $2 \mathrm{~b}$ ).

TABLE I

DUBLIN SCENARIO SIMULATION PARAMETERS

\begin{tabular}{|l|l|l|}
\hline \multicolumn{3}{|c|}{ Dublin Scenario Simulation Parameters } \\
\hline \hline Site and Sector & Inter-site distance & $800 \mathrm{~m}$ \\
\hline \hline \multirow{3}{*}{ Channel } & NLOS exponential path loss factor & 3.9 \\
& NLOS fixed path loss factor & $10^{-2.1}$ \\
& LOS exponential path loss factor & 2.2 \\
& LOS fixed path loss factor & $10^{-3.4}$ \\
& Shadow fading standard deviation & 6 \\
& Shadow fading mean & 0 \\
\hline
\end{tabular}

To provide a baseline, Figure 3 shows the proportional fair rate allocation with SISO receivers and non-adaptive roundrobin scheduling. For comparison, results are also shown when a fixed tilt angle of $8^{\circ}$ is used. Figure 3(a) shows the tilt angle adaptation, which quickly settles on a configuration that improves the sum-log-throughput objective function by $22 \%$ compared with the fixed tilt angle. From the cumulative distribution function (CDF) in Figure 3(b) it can be seen the user throughputs are also significantly increased, with the median throughput increased by almost a factor of 4 compared to use of fixed angles.

Figure 4 shows the corresponding CDF of the user throughputs when SIMO links with MMSE detection are used. Data 


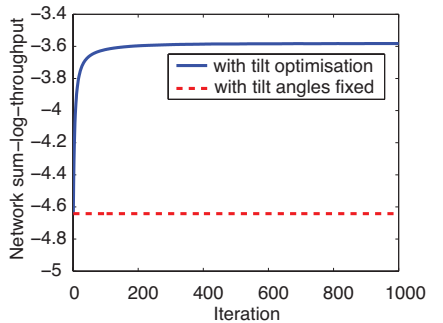

(a) Normalised Sum-log-throughput

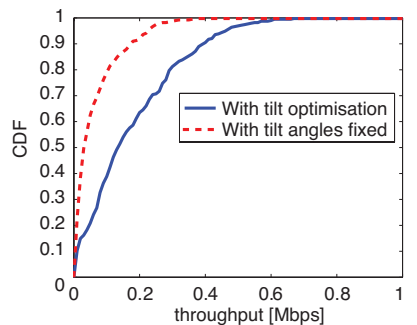

(b) CDF of user throughputs.
Fig. 3. Proportional fair rate allocation, Dublin example. SISO receivers and round-robin scheduling. For comparison, results are also shown when a fixed tilt angle of $8^{\circ}$ is used (indicated by dashed lines).

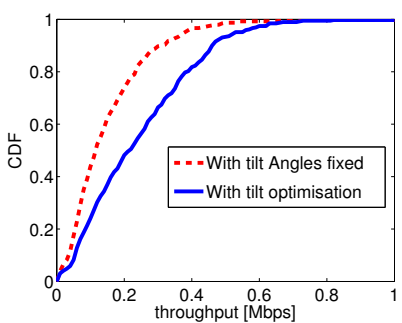

(a) with fast fading

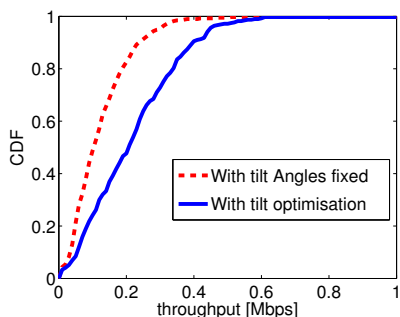

(b) without fast fading
Fig. 4. User throughput CDFs with SIMO links and MMSE detection, Dublin example. Fast fading is modelled by generating 300 samples using the 3GPP typical urban channel model, where the speed of the mobile user and carrier frequency are $3 \mathrm{~km} / \mathrm{h}$ and $2 \mathrm{GHz}$ respectively.

is shown with and without flat fading. As might be expected, the use of MMSE detection yields significant improvements in the user throughputs. The throughput gains achieved by tilt optimisation can be compared for SISO links and for SIMO links with an optimal LMMSE detector by comparing Figures $3 \mathrm{~b}$ and 4 . The gain in the mean user throughput achieved by tilt optimisation is decreased from $83.07 \%$ to $67.42 \%$ when MMSE detection is employed. However, the gain in the sum-log-rate (which is the objective function of optimisation $P$ ) only changes from $22.29 \%$ to $22.00 \%$. That is, while MMSE detection enhances inter-cell interference mitigation, tilt optimisation can still yield significant improvements in network capacity.

We can investigate this behaviour in more detail as follows. Let

$$
\epsilon_{u}=\frac{\max _{c \in \mathcal{B} \backslash\{b(u)\}}\left\{\hat{H}_{u}\left(\theta_{c}\right) 10^{S_{c, u} / 10}\right\}}{\sum_{c \in \mathcal{B} \backslash\{b(u)\}} \hat{H}_{u}\left(\theta_{c}\right) 10^{S_{c, u} / 10}}
$$

be the ratio of the largest interferer to the total interference experienced by a user $u$. The CDF of $\epsilon$ for the Dublin example is shown in Figure 5(a). It can be seen that approximately $40 \%$ of users have $\epsilon$ values less than 0.5 i.e. for $40 \%$ of users the the strongest interferer power is less than the sum of the power of the other interferers. Figure 5(b) shows the corresponding spatial distribution of $\epsilon$. It can be seen that the strongest interferer is dominant at the edge of antenna sectors and along the nulls of the sector antennas. However, the intensity of the strongest interferer decreases along the edges of the base station coverage area and alongside the

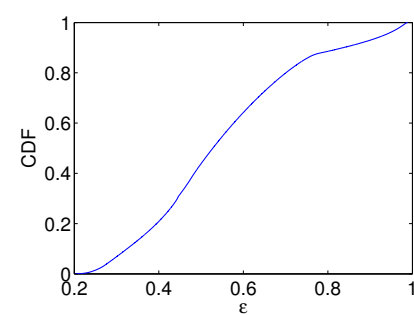

(a)

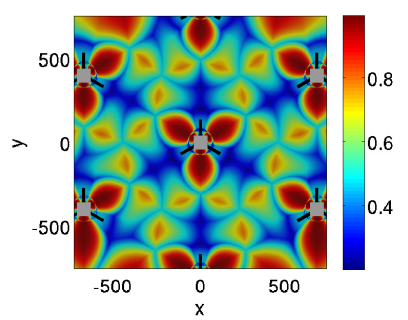

(b)
Fig. 5. Contribution of the strongest interference to the total Interference: (a) cumulative distributed function of $\epsilon$ in the central BS coverage area. (b) Distribution of $\epsilon$ relative to the user positions.

TABLE II

USER THROUGHPUT GAINS DUE TO TILT ANGLE OPTIMISATION FOR BOTH SISO AND SIMO LINKS AND VS $\epsilon$, DUBLIN EXAMPLE.

\begin{tabular}{|l||l|l|}
\hline \multicolumn{1}{|c||}{} & \multicolumn{2}{c|}{ Mean Throughput Gain [\%] } \\
\cline { 2 - 3 } & SISO & SIMO \& MMSE Detection \\
\hline \hline $0 \leq \epsilon_{u} \leq .5$ & 54.5 & 52.3 \\
$.5<\epsilon_{u} \leq .8$ & 128 & 85.99 \\
$.8<\epsilon_{u} \leq 1$ & 147 & 91.99 \\
\hline $0 \leq \epsilon_{u} \leq 1$ & 83.07 & 67.42 \\
\hline
\end{tabular}

antennas. Table II details the throughput gains achieved by tilt angle optimisation for both SISO and SIMO links and for users with different $\epsilon$ ratios. It can be seen that the throughput gain obtained by tilt angle optimisation for users with $\epsilon>.5$ is reduced when SIMO links are used. However, the gain is similar for both SISO and SIMO links for users with $\epsilon \leq .5$, once MMSE post processing is applied, and as noted above this consists of approximately $40 \%$ of users.

In summary, although the mean user throughput is improved for both fixed and optimal tilt angles for SIMO links with MMSE, tilt optimisation can still yield considerable performance gains.

\section{Multi-USER Diversity AND NARRowbAND SINR ALLOCATION}

Both time and frequency domain diversity in LTE can be exploited to develop Quality of Service (QoS) orientated Time and Frequency Domain (TD and FD) scheduling techniques. In particular, proportional fair scheduling and the use of multiuser diversity can improve user SINRs, specifically for the fixed users as they are less sensitive to the delay of the Channel Quality Indicator (CQI) reports.

\section{A. Proportional Fair Adaptive Transmission Scheduling}

The minimum resolution for frequency domain scheduling is called the Physical Resource Block (PRB). We indicate the number of resource blocks by $n$ and the number of users by $u$. Following [14] and [15], in the TD Proportional Fair (TD-PF) scheduler, users are first ranked based on their past average throughput $(T[u])$ at transmission time interval $t$ by the following metric:

$$
M_{T D-P F}=\frac{\hat{D}[u]}{T[u]}
$$


Where $\hat{D}[u]$ is the wideband throughput. Thereafter, $N_{u}$ users with the highest priority are assigned to the FD scheduler at each time interval, where $N_{u}$ depends on the number of the resource blocks and potential signalling constraints [14]. Moreover, it should be noted that number of users served by each cell may be considerably greater than $N_{u}$. We look at two types of frequency domain schedulers. First, FD-PF where the $N_{u}$ user allocations to the appropriate PRBs are given by:

$$
M_{F D-P F}[n, u]=\frac{\hat{d}[n, u]}{T[u]}
$$

with $\hat{d}[n, u]$ is the estimated achievable throughput for user $u$ and resource block $n$ calculated base on the CQI report for the particular PRB and assuming equal power per PRB. The second FD scheduler is Carrier over Interference to Average (CoItA) where allocations to the resource blocks are defined by:

$$
M_{F D-P F}[n, u]=\frac{\hat{\gamma}[n, u]}{\sum_{u=1}^{u=N} \hat{\gamma}[n, u]}
$$

with $\hat{\gamma}[n, u]$ is an estimation of SINR on the $n^{\text {th }}$ PRB and $u^{t h}$ user. As user SINRs on each RB differ due to fading characteristics across the bandwidth, $\mathrm{RB} n$ is assigned to the user with the best fading gain and as fading is independently distributed between users, all users have the same probability of accessing the RB.

\section{B. Performance Evaluation}

In this section we consider two scenarios : (i) where proportional fair scheduling is applied on a network with SISO links, and (ii) where proportional fair scheduling is applied on a network with SIMO/LMMSE reception.

1) PF Scheduling for SISO Links: We modelled frequency selective fading by independent flat fading events per subchannel with a user speed of $3 \mathrm{Km} / \mathrm{h}$. The distribution of fading events among users are assumed independent and identical. The number of PRBs across the channel bandwidth is 50 with $200 \mathrm{KHz}$ bandwidth assigned to each PRB. The number of prioritised users in the TD-PF scheduler at each time interval is 10 with 400 time slots of $1 \mathrm{~ms}$ duration.

Figure 6 shows the CDF of the user throughputs when proportional fair scheduler is used in the Dublin network described above in Section IV-B. It can be seen that, as might be expected, the user throughputs are improved for both the baseline (fixed tilt angles) and the optimum tilt angles compared to those of Figure 3(b) where round-robin scheduling is used. When tilt angle optimisation is used, the mean throughput increases by $65 \%$, compared with an increase of $83 \%$ when round-robin scheduling is employed. However, the gain in the sum-log-rate (the optimisation objective) reduces only very slightly from $22 \%$ to $20.9 \%$ when a proportionalfair (FD-PF) scheduler is used.

2) PF Scheduling for SIMO/LMMSE Reception: In this section, we consider joint operation of SIMO link/LMMSE detection and narrowband scheduling in the Dublin network described in Section IV-B. Herein scheduling is adapted based

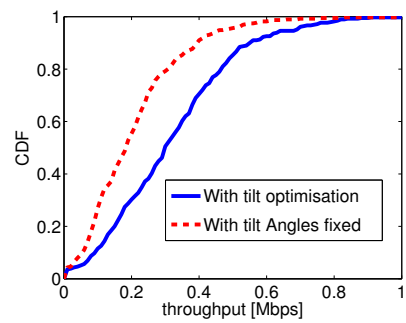

(a) FD-PF

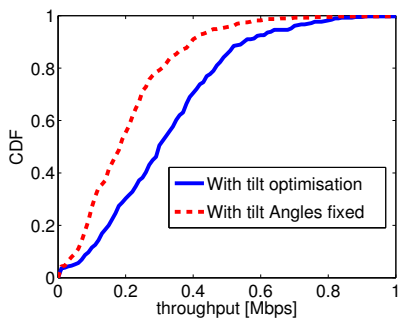

(b) FD-CoItA
Fig. 6. CDF of user throughputs when using proportional fair scheduling, Dublin example.

on estimated post-processing SINR and throughput values. In this section we assume CQI reports can be provided in terms of post-processing SINRs and with minimum processing time while perfect channel information is available at the receiver. We used the same channel model as that of the previous scenario. Figure 7 shows the $\mathrm{CDF}$ of user throughputs when adaptive scheduling and LMMSE detection are jointly applied. It can be seen that user throughputs are improved for both baseline (fixed tilt angles) and optimum tilt angles compared to those of Figures 3(b) ( round-robin scheduling for SISO links), 4 (round-robin scheduling for SIMO links and post-processing), and 6 (proportional fair scheduling for SISO links). When tilt angle optimisation is used the case of FD-CoItA scheduler, the gain in mean throughput is reduced to $47 \%$ compared to $83 \%$ mean throughput gain of round-robin scheduling and SISO links. However, the gain in sum-log-rate is only reduced to $19 \%$ compared to $22 \%$ gain of round-robin scheduling and SISO links. In the case of FD-PF scheduler, mean user throughput gain due to tilt optimisation is $51 \%$ and the gain in sum-log-rate objective is $21 \%$.

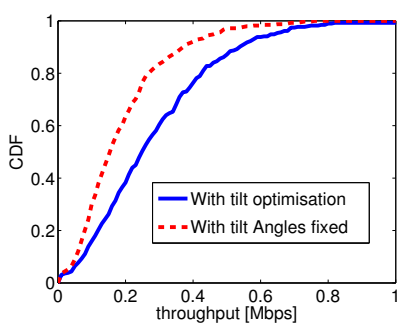

(a) FD-PF

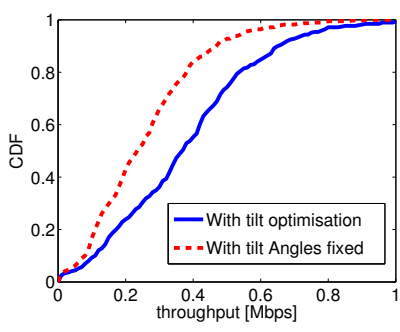

(b) FD-CoItA
Fig. 7. CDF of user throughputs when using proportional fair scheduling based on estimated LMMSE post processing SINRs, Dublin example.

\section{CONCLUSIONS}

In this paper we investigate the role of tilt angle adjustment in next generation LTE networks where SIMO receivers and adaptive OFDM/TDMA transmission scheduling may additionally be used to mitigate interference. We present detailed performance measurements when (i) optimal fair tilt angle adjustment is applied in combination with SIMO receivers using Linear Minimum Mean Square Error (LMMSE) detection to mitigate interference and when (ii) tilt angle adjustment is applied in combination with proportional fair OFDM 
transmission scheduling which adapts the transmission rate per subcarrier (narrow-band rate allocation). We find that even when SIMO/LMMSE reception and adaptive transmission scheduling are used to mitigate interference, tilt angle adjustment still offers the potential for significant performance gains, namely increases in mean user throughput of more than $65 \%$ and improvements in the network sum-log rate of greater than $20 \%$. Moreover, we show that even when adaptive narrowband scheduling is jointly used with SIMO/LMMSE reception, tilt angle adjustment offers $51 \%$ gain in mean user throughput and $19 \%$ gain in the network sum-log-rate. That is, there appears to exist a useful degree of decoupling between tilt angle adjustment and the details of receiver and transmitter design which has the potential to greatly simplify network design. Of particular interest is that these performance gains are achieved even when the tilt angle adjustment assumes basic single antenna receivers and non-adaptive round-robin frequency scheduling.

\section{REFERENCES}

[1] I. Forkel, A. Kemper, R. Pabst, and R. Hermans, "The effect of electrical and mechanical antenna down-tilting in umts networks," in $3 G$ Mobile Communication Technologies, 2002. Third International Conference on (Conf. Publ. No. 489), pp. 86-90, IET, 2002.

[2] F. Athley and M. Johansson, "Impact of electrical and mechanical antenna tilt on lte downlink system performance," in Vehicular Technology Conference (VTC 2010-Spring), 2010 IEEE 71st, pp. 1-5, IEEE, 2010.

[3] 3rd Generation Partnership Project; Technical Specification Group Radio Access Network; Evolved Universal Terrestrial Radio Access (EUTRA); LTE physical layer, general description (Release 11), 2012.

[4] B. Partov, D. J. Leith, and R. Razavi, "Utility fair optimisation of antenna tilt angles in lte networks," 2013. To be appeared in IEEE Transactions/ACM on Networking. Available at http://arxiv.org/abs/1310.1015.

[5] E. Benner and A. Sesay, "Effects of antenna height, antenna gain, and pattern downtilting for cellular mobile radio," Vehicular Technology, IEEE Transactions on, vol. 45, no. 2, pp. 217-224, 1996.

[6] O. Yilmaz, S. Hamalainen, and J. Hamalainen, "Analysis of antenna parameter optimization space for 3gpp lte," in Vehicular Technology Conference Fall (VTC 2009-Fall), 2009 IEEE 70th, pp. 1-5, IEEE, 2009.

[7] H. Eckhardt, S. Klein, and M. Gruber, "Vertical antenna tilt optimization for lte base stations.," in VTC Spring, pp. 1-5, IEEE, 2011.

[8] R. Razavi, S. Klein, and H. Claussen, "A fuzzy reinforcement learning approach for self optimization of coverage in lte networks," Bell Lab. Tech. J., vol. 15, pp. 153-175, Dec. 2010.

[9] A. Temesváry, "Self-configuration of antenna tilt and power for plug \& play deployed cellular networks," in Wireless Communications and Networking Conference, 2009. WCNC 2009. IEEE, pp. 1-6, IEEE, 2009.

[10] G. Calcev and M. Dillon, "Antenna tilt control in cdma networks," in Proceedings of the 2nd annual international workshop on Wireless internet, p. 25, ACM, 2006.

[11] A. Eisenblatter and H. Geerdes, "Capacity optimization for umts: Bounds and benchmarks for interference reduction," in Personal, Indoor and Mobile Radio Communications, 2008. PIMRC 2008. IEEE 19th International Symposium on, pp. 1-6, IEEE, 2008.

[12] 3rd Generation Partnership Project; Technical Specification Group Radio Access Network; Evolved Universal Terrestrial Radio Access (E-UTRA); Further advancements for E-UTRA physical layer aspects (Release 9), 2010.

[13] Universal Mobile Telecommu nications System (UMTS); Spatial channel model for Multiple Input Multiple Output (MIMO) simulations (Release 11), 2012.

[14] A. Pokhariyal, K. I. Pedersen, G. Monghal, I. Z. Kovacs, C. Rosa, T. E. Kolding, and P. E. Mogensen, "Harq aware frequency domain packet scheduler with different degrees of fairness for the utran long term evolution," in Vehicular Technology Conference, 2007. VTC2007-Spring. IEEE 65th, pp. 2761-2765, IEEE, 2007.

[15] G. Mongha, K. I. Pedersen, I. Z. Kovacs, and P. E. Mogensen, "Qos oriented time and frequency domain packet schedulers for the utran long term evolution," in Vehicular Technology Conference, 2008. VTC Spring 2008. IEEE, pp. 2532-2536, IEEE, 2008. 